\title{
Thermomechanic-electrical coupling in phospholipid monolayers near the critical point
}

\author{
D. Steppich, ${ }^{1}$ J. Griesbauer, ${ }^{1,2, *}$ T. Frommelt, ${ }^{1}$ W. Appelt, ${ }^{1}$ A. Wixforth, ${ }^{1}$ and M. F. Schneider ${ }^{2, \dagger}$ \\ ${ }^{1}$ Experimental Physics I, University of Augsburg, Universitätstsr. 1, D-86159 Augsburg, Germany \\ ${ }^{2}$ Mechanical Engineering, Boston University, 110 Cummington Street, Boston, Massachusetts 02215, USA
}

(Received 22 January 2010; revised manuscript received 24 March 2010; published 15 June 2010)

\begin{abstract}
Lipid monolayers have been shown to represent a powerful tool in studying mechanical and thermodynamic properties of lipid membranes as well as their interaction with proteins. Using Einstein's theory of fluctuations we here demonstrate that an experimentally derived linear relationship both between transition entropy $S$ and area $A$ as well as between transition entropy and charge $q$ implies a linear relationships between compressibility $\kappa_{T}$, heat capacity $c_{\pi}$, thermal expansion coefficient $\alpha_{T}$, and electric capacity $C_{T}$. We demonstrate that these couplings have strong predictive power as they allow calculating electrical and thermal properties from mechanical measurements. The precision of the prediction increases as the critical point $T_{C}$ is approached.
\end{abstract}

DOI: 10.1103/PhysRevE.81.061123

PACS number(s): 05.70.Np, 68.18.-g, 68.35.Md

\section{INTRODUCTION}

Lipid monolayers have been of major interest for a variety of reasons. They contain many interesting aspects of physics in two dimensions, allowing us to study intermolecular interactions between lipids and/or proteins and mimic one leaflet of a biomembrane in which proteins can be incorporated fairly easy. Moreover, in contrast to most thin film physics experiments, the majority of measurements can be performed without a vast amount of technical equipment and preparation [1]. The prepared monomolecular film can then be transferred on a solid support for further investigation [2]. Most macroscopic conditions (pressure, temperature, concentration, etc.) are easily accessible and thermodynamic properties can be measured straight forwardly. Furthermore, using the appropriate thermodynamic relationships, the measured quantities can be related to the heat of transition and several response functions like compressibility, thermal expansion coefficient, electrical capacity, and, as will be shown in this paper, even heat capacity.

As was demonstrated earlier for lipid vesicles [3], there exists a linear relationship between the excess isothermal compressibility $\Delta \kappa_{\mathrm{T}}$ and the heat capacity $\Delta c_{p}$ at constant pressure,

$$
\Delta \kappa_{T} \propto \Delta c_{P} .
$$

This relationship implies a pronounced maximum of the compressibility in the phase transition regime, following the well-known profile of $\Delta c_{p}$. However, there is no physical explanation of the origin of this relationship to date.

\footnotetext{
*Corresponding author for electrical measurements and experiments: University of Augsburg Experimentalphysik 1 Universitätsstr. 186159 Augsburg (Germany);

Josef.Griesbauer@physik.uni-augsburg.de

†Corresponding author: Boston University Mechanical Engineering, 110 Cummington Street, Boston, MA, 02215 (USA); mfs@bu.edu
}

Here, we show that the experimentally established proportionality between entropy and area and entropy and charge in combination with Einstein's theory of fluctuations consequently leads to linear relationships between compressibility $\kappa_{T}$, heat capacity $c_{\pi}$, thermal expansion coefficient $\alpha_{T}$, and electric capacity $C_{T}$. This allows to calculate the corresponding heat capacity of lipid monolayers which is shown to be in good agreement to calorimetric data found for lipid vesicle suspensions [3].

\section{MATERIAL AND METHODS}

The phospholipids DMPC (1,2-Dimyristoyl-snGlycero-3-Phosphocholine), DPPC (1,2-Dipalmitoyl-snGlycero-3-Phosphocholine), DMPG (1,2-Dimyristoyl-snGlycero-3-[Phospho-rac-(1-glycerol)]), and DOTAP (1,2Dioleoyl-3-Trimethylammonium-Propane) were purchased from avanti polar lipids (Al, USA). Lipids were dissolved in pure chloroform and used without further purification. For measuring pressure-area and potential-area isotherms we used film balances of Nima Technologies (Coventry, England), which were partly equipped with a Kelvin Probe Surface Potential of TREK (New York, USA) for measuring the surface potential of the lipid films. $500 \mathrm{ml}$ of double distilled water is filled into a trough and the lipid-chloroform solution is spread onto the water surface. After evaporation of the solvent the film was compressed with a speed of approximately $3 \mathrm{~cm}^{2} / \mathrm{min}$ or when accounting for the through size $0.4 \times 10^{-16} / \mathrm{min}$ per lipid. At the same time the lateral pressure of the surface is measured using a Wilhelmy plate and the surface potential is recorded if desired. To calculate the compressibility we subdivided the pressure-area curve in three sections and fitted each section by a polynomial function of seventh degree or higher to assure only minimal deviations (less than the experimental error bar) from the experimental curve. The fits were then merged together to reach continuously differentiable plots. For conserving the stronger fluctuations of the surface potential curves, numerical derivations were made for calculation of the electrical capacity. 

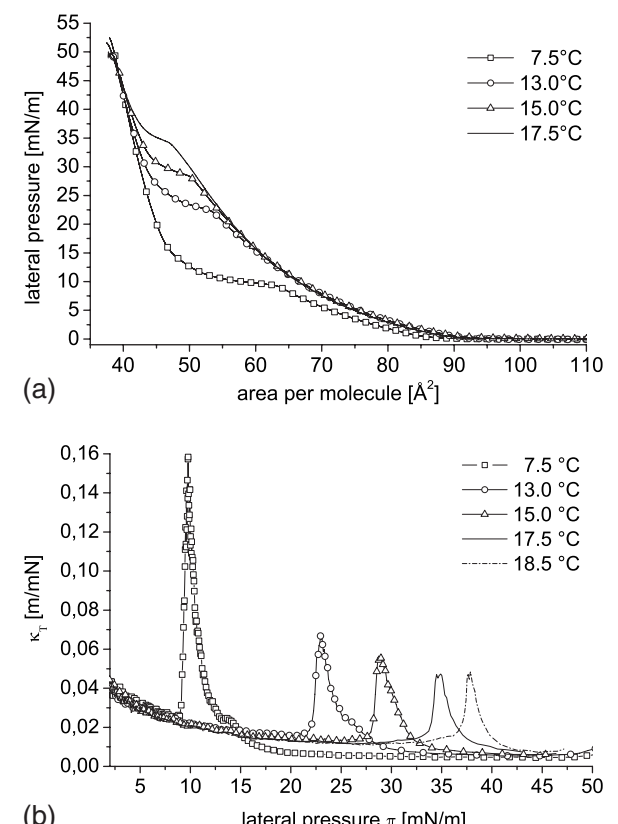

(b)

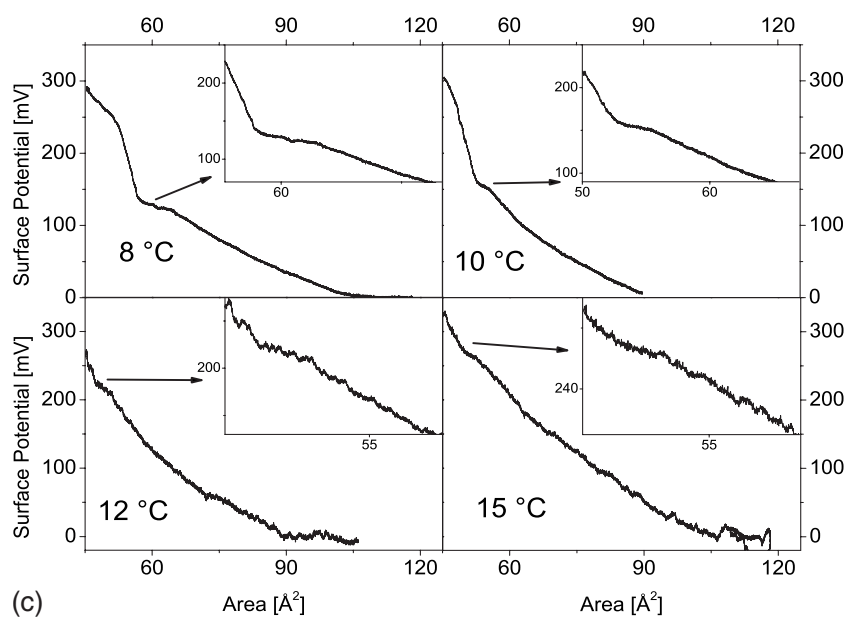

FIG. 1. [(a) and (b)] DMPC pressure-area isotherms measured for different temperatures. For the sake of clarity only four isotherms are shown. From the raw data of the upper panel, the isothermal compressibility is calculated, showing a pronounced maximum during the phase transition regime (lower panel). (c) DMPC potential-area isotherms measured for different temperatures. The region around phase transition is plotted in a separate inset for every temperature, demonstrating the disappearance of the plateau with increasing temperature.

\section{RESULTS AND DISCUSSION}

\section{A. Experimental results}

\section{Linear relationship among area, entropy, and charge}

In Figs. 1(a) and 1(b) a set of pressure-area isotherms is shown along with the calculated isothermal compressibility [4],

$$
\kappa_{T}=-\left.\frac{1}{A} \frac{\partial A}{\partial \pi}\right|_{T} .
$$

Here, $A$ is the area per molecule and $\pi$ the lateral pressure, which might not be mistaken with the global pressure $p$ as
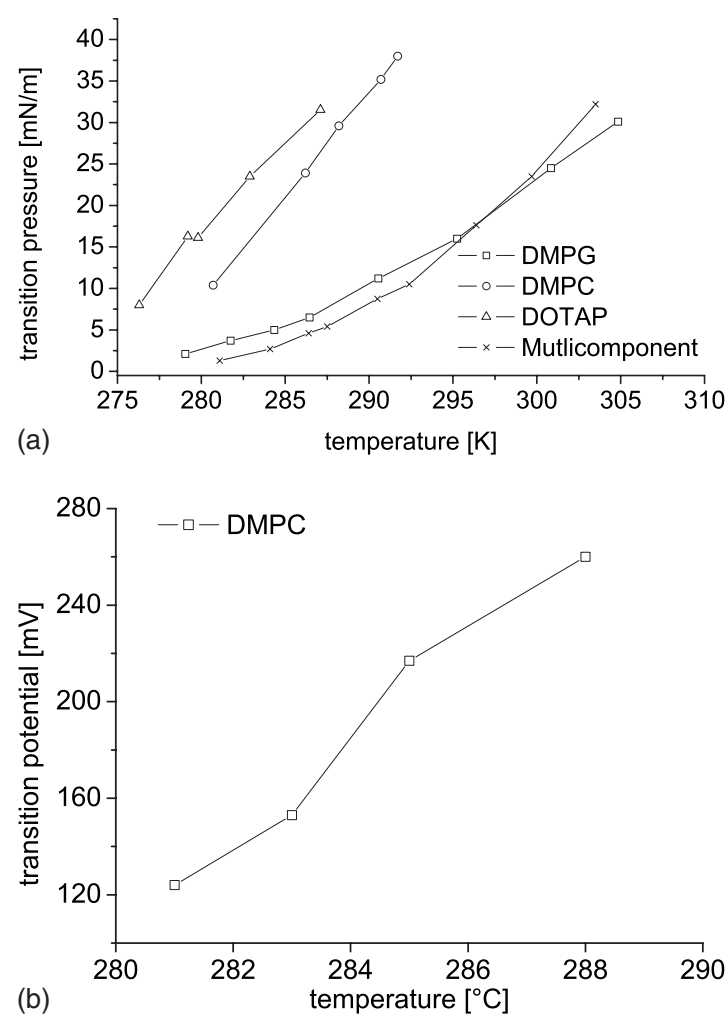

FIG. 2. (a) Plot of the transition pressure $\pi_{t}$ of the phospholipids DOTAP, DMPC, DMPG, and a multicomponent system consisting of $40 \mathrm{~mol} \%$ DMPC, $40 \mathrm{~mol} \%$ DMPG, and $20 \mathrm{~mol} \%$ DPPC as a function of temperature $T$. A linear relationship $\pi_{t}(T)$ is clearly conserved for all systems. (b) Transition Potentials $\Psi_{t}$ of DMPC as a function of temperature. Clearly, the surface potential $\Psi_{t}(T)$ increases linearly with temperature in transition regime.

denoted in $c_{p}$. Additionally a set of surface potential-area isotherms $(\Psi-A)$ are plotted in Fig. 1(c) to demonstrate their similarity with the typical pressure-area isotherms [Fig. 1(a)] especially in the phase transition regime, whereas this is a well-known behavior [5-7].

From Figs. 1(a) and 1(b), the change in phase transition pressure $\pi_{t}$ as a function of temperature $T$ can be calculated and is plotted in Fig. 2(a). A linear relationship $\pi_{t}(T)$ as has been reported before $[8,9]$ can be resolved. Interestingly the same behavior is resembled for the transition potentials $\Psi_{t}(T)$ shown in Fig. 2(b) indicating a more general character of the observed linearity, with a coupling of the thermodynamic observables studied.

When examining the pressure-area data over a wider temperature range, one finds that this linear relationship is more pronounced in the high-temperature regime as the critical point is approached but departs clearly for lower temperature [Fig. 2(a)]. This has been found earlier, e.g., by Albrecht et al. [8] and Hato et al. [10]. Qualitatively, the observed linearity seems to be independent of the nature of the lipid head group and could be reproduced for different lipids (DMPC, DMPG, and DOTAP) and lipid mixtures of three different lipids [Fig. 2(a)]. The linear character is further supported by recent results [11] where the same qualitative relationship was found for a whole set of synthesized glycolipids containing different size sugar (lactose) headgroups and no phosphoric acid. 


\section{B. Theoretical results}

The last section has experimentally shown that the change in phase transition pressure $\pi_{t}$ and potential $\Psi_{t}$ with temperature $T$ occurs linear in the regime of the critical point. As a consequence, the change in area, charge, and entropy during the phase transition are linearly related according to the Clausius-Clayperon equation [5]:

$$
\begin{aligned}
& \left.\frac{\partial \pi_{t}}{\partial T}\right|_{\text {Trans }}=\frac{\Delta S}{\Delta A}=\text { const }=B_{\pi}, \\
& \left.\frac{\partial \Psi_{t}}{\partial T}\right|_{\text {Trans }}=\frac{\Delta S}{\Delta q}=\text { const }=B_{\Psi}
\end{aligned}
$$

and therefore

$$
\begin{aligned}
& \Delta S=B_{\pi} \Delta A, \\
& \Delta S=B_{\Psi} \Delta q .
\end{aligned}
$$

For the transition pressures this relationship seems to be well conserved and becomes more accurate in the vicinity of the critical point.

When approaching the critical point the double minimum of the free energy gradually disappears and changes into a single rather flat minimum. In the near vicinity the potential can therefore be approximated by $S=S_{0}+\sum \partial S / \partial x_{i} x_{i}$ $+\sum \partial^{2} S / \partial x_{i} \partial x_{j} x_{i} x_{j}$. The corresponding fluctuations are

$$
\left\langle x_{i} x_{j}\right\rangle=-k_{B}\left(\frac{\partial^{2} S}{\partial x_{i} x_{j}}\right)^{-1} .
$$

Here, $x_{i}$ and $x_{j}$ are extensive thermodynamic variables (area, charge, particle number, etc.), $k_{B}$ is the Boltzmann constant, and $S\left(x_{i}, x_{j}\right)$ is the proper thermodynamic (entropy) potential. We have recently shown $[12,13]$ that $S$ of the monolayer can indeed be considered as decoupled from the bulk, which holds for adiabatic properties as well as for reversible fluctuations down to the nanoscale. Now using Eq. (4), one gets

$$
\begin{gathered}
\left\langle\delta A^{2}\right\rangle=A k_{B} T \kappa_{T}, \\
\left\langle\delta q^{2}\right\rangle=\left.k_{B} T \frac{\partial q}{\partial \Psi}\right|_{T, A}=k_{B} T C_{T}, \\
\left\langle\delta S^{2}\right\rangle=k_{B} c_{\pi},
\end{gathered}
$$

where $C_{T}$ is the electrical capacity. For the fluctuation correlations between area and entropy

$$
\begin{gathered}
\langle\delta S \delta A\rangle=A k_{B} T \alpha_{\pi}, \\
\langle\delta S \delta q\rangle=\left.k_{B} T \frac{\partial q}{\partial T}\right|_{\Psi, A} .
\end{gathered}
$$

In other words, area fluctuations are proportional to the isothermal compressibility $\kappa_{T}$, entropy fluctuations are proportional to the isobaric heat capacity $c_{\pi}$ and charge fluctuations are proportional to the electrical capacity $C_{T}$. The correlated fluctuations are proportional to the isobaric area expansion coefficient $\alpha_{\pi}$ or the derivation of the charge $\partial q /\left.\partial T\right|_{\Psi, A}$. We like to note here that while Eqs. (5) and (6) are accepted textbook representations, the physically interpretation of a fluctuating entropy potential [Eqs. (6a) and (6b)] is physically not clear to these authors.

Under the assumption that the linear relationship in Eq. (3) holds also for small fluctuations $(\Delta \sim \delta)$ one finds using Eqs. (3), (5), and (6):

$$
\begin{aligned}
& \Delta c_{\pi}=B_{\pi}^{2} A T \Delta \kappa_{T}, \\
& \Delta c_{\pi}=B_{\pi} A T \Delta \alpha_{\pi}, \\
& \Delta c_{\pi}=B_{\Psi}^{2} T \Delta C_{T},
\end{aligned}
$$

and therefore

$$
\Delta \kappa_{T} \propto \Delta \alpha_{\pi} \propto \Delta c_{\pi} \propto \Delta C_{T} .
$$

Partly, for thermal and mechanical properties, this was already put forward by Heimburg [Eq. (3)] for lipid vesicle suspensions. He demonstrated that one can assume $\Delta A$ $\sim \delta A$ when the "excess" heat capacity $\Delta c_{\pi}$ and compressibility $\Delta \kappa_{T}$ are proportional for a wide $T$ range, the latter of which has been derived experimentally.

Equation (10) states, that the excess response functions exhibit a simple linear relationship, which predicts, according to Fig. 1, a pronounced maximum in area expansion coefficient, the electrical capacity, and the heat capacity close to the phase transition regime.

In the following section we will compare our theoretical predictions with our experimental results.

\section{Discussion}

\section{Coupling between thermal and elastic properties during phase transition}

In Fig. 3(a) the area per molecule as a function of $T$ is plotted for three different $\pi$ values. When calculating the corresponding $\alpha_{\pi}$ using $\alpha_{\pi}=1 / \bar{A} \partial A /\left.\partial T\right|_{\pi}$ [Fig. 3(b)] a pronounced maximum with a shoulder on the low-temperature site and a steep decrease in the liquid expanded phase is found. This is very similar to the experimental results of $\kappa_{T}$ [Fig. 1(b)], where we also resolve a shoulder toward the liquid condensed phase and a steep change in $\kappa_{T}$ when leaving the liquid expanded phase. The enormous predictive power of the Eqs. (7)-(10) can be demonstrated by calculating the thermal expansion from the compressibility using $\Delta \kappa_{T}=-\Delta \alpha_{\pi} / B_{\pi}$. When taking for example $\kappa_{T}^{\max }$ at $13{ }^{\circ} \mathrm{C}$ $(285 \mathrm{~K})$ and $23 \mathrm{mN} / \mathrm{m}$ [Fig. 1(b)], we find $\alpha_{\pi} \approx 0,4 \mathrm{~K}^{-1}$, which is close to $\alpha_{\pi}^{\max }=0,35 \mathrm{~K}^{-1}$ (at $13.6{ }^{\circ} \mathrm{C}$ ) extracted from the expansion measurement directly [Eq. (3b)]. This quantitative and qualitative agreement indicates that the extrapolation from $\Delta A$ to area fluctuations $\delta A$ used to derive Eq. (10) is well justified.

\section{Coupling between electrical and elastic properties during phase transition}

As $\Psi$ was measured for DMPC, the electrical capacity $C_{T}$ can be calculated according to $C_{T}=d q / d \Psi=$ 

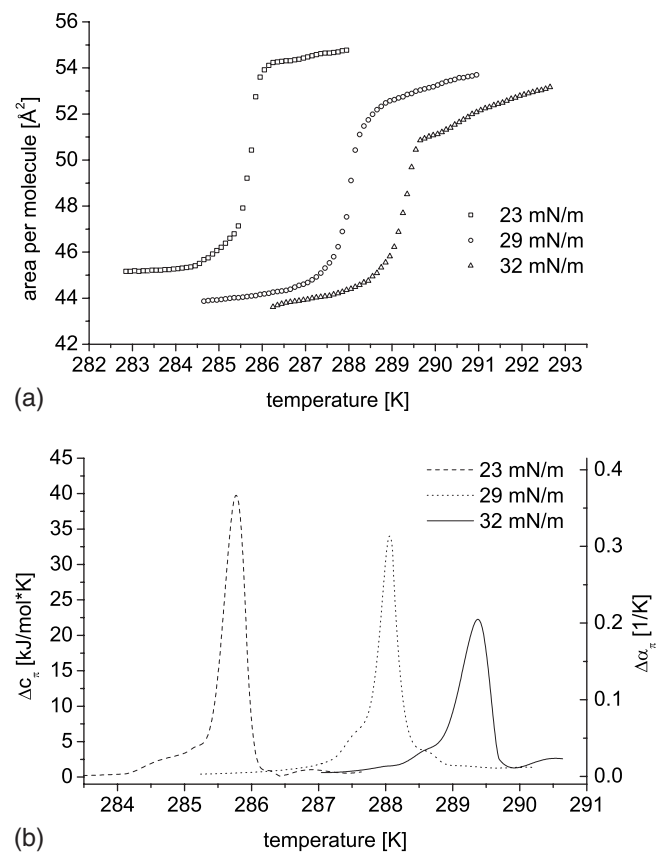

(b)

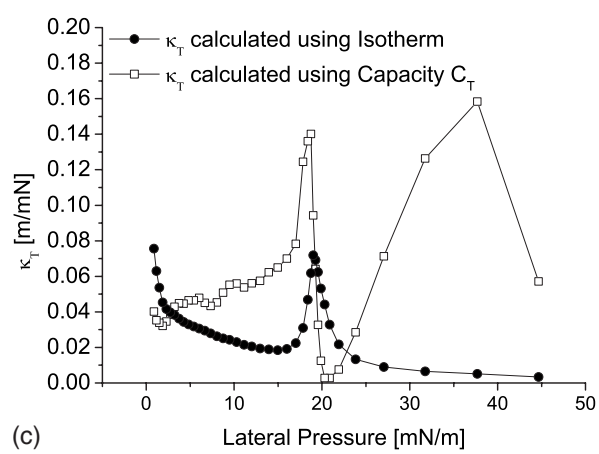

FIG. 3. (a) By keeping the lateral pressure constant and varying the temperature, the area per molecule can be measured as a function of temperature. During the phase transition, the slope of the graph significantly increases. (b) From (a) the isobaric expansion coefficient $\alpha_{\pi}$ can be calculated. Similar to the heat-capacity profiles, calculated using Eq. (11), it displays a maximum in the phase transition regime. The graphs display qualitatively and quantitatively (heat of transition) very similar features as the heat capacity obtained by DSC for giant unilamellar vesicles. (c) Our relations [Eq. (10)] allow to directly compare the electrical capacity $C_{T}$ with the mechanical compressibility $\kappa_{T}$ at $8{ }^{\circ} \mathrm{C}$. The compressibility as calculated from the electrical capacity $C_{T}$ using Eqs. (7)-(9) is plotted in the same graph as the actual measured (using pressure-area isotherms) compressibility. Both curves show the typical fingerprints of a phase transition.

$-\partial A /\left.\partial \Psi\right|_{q, I} \partial \pi /\left.\partial \Psi\right|_{A, T}$ (where we have used the Maxwell relation: $\left.-\partial A /\left.\partial \Psi\right|_{q, T}=: \partial q /\left.\partial \pi\right|_{A, T}\right)$. By using $\Psi_{t}(T)$ to determine $B_{\Psi}$ from a linear fit [Eq. (2b)], $C_{T}$ can directly be compared to $\kappa_{T}$. Although the correlation is more pronounced in Figs. 3(a) and 3(b), the coupling is clearly visible in Fig. 3(c) as well.

\section{Heat capacity of lipid monolayers}

The isobaric heat capacity $c_{\pi}$ [compare to $c_{p}$ as measured by differential scanning calorimetry (DSC) for small unila- melar lipid vesicles (SUVs)] of lipid monolayer is not directly experimentally accessible [12]. However using the relation shown in Eq. (10) we are now able to calculate $c_{\pi}(T)$ from $\alpha_{\pi}[14]$.

The result plotted in Fig. 3(b) contains a pronounced maximum, which qualitatively resembles the heat-capacity profile of a lipid vesicle suspension. Remarkably, considering the calculated monolayer heat-capacity profile at 32 $\mathrm{mN} / \mathrm{m}$ both the half width at half height (HWHH) as well as the absolute value of the maximum in $\Delta c_{\pi}(T)$ agree within $20 \%$ with the heat-capacity profile of lipid SUVs of the same type [15]. Surprisingly, even the shoulder in the liquid ordered (gel) phase known from DSC measurements is found in our monolayer experiments. We believe that further theoretical investigations on this observation might give insight on topological and pure in plane effects on the heat-capacity profile of lipid suspensions such as SUVs since lipid monolayers simply cannot undergo morphological transitions as opposed to lipid vesicles [16-19].

\section{Role of the critical point}

The general character of the linear relationship between $\alpha_{\pi}, \kappa_{T}, c_{\pi}$, and $C_{T}$ calls for a more profound understanding of the origin of this linear relationship. Obviously, the character of the first-order transition (e.g., plateau width) becomes increasingly weaker as the temperature rises [Fig. 1(a)], a typical fingerprint for the existence of a critical point. The same holds when looking at the area jump in Fig. 3(a): as the pressure is increased toward the critical point the abrupt change in area decreases. Indeed, it has been stated, that the lipid monolayer phase transition is only of weak first order in the vicinity of a second-order transition [20,21].

In summary one can say, that all experiments indicate the trend toward a critical point as the temperature is increased. Since the symmetry between liquid expanded and liquid condensed phase remains beyond the critical point, the firstorder transition must converge into a second-order transition (tricritical point) [8]. At this point the Clausius-Clayperon equation for second-order phase transition states [5]:

$$
\frac{\partial \pi}{\partial T}=\frac{\Delta c_{\pi}}{A T \Delta \alpha_{\pi}}
$$

or

$$
\frac{\partial \Psi}{\partial T}=\frac{\Delta c_{\pi}}{T \Delta C_{T}}
$$

The vicinity of a critical point therefore implies $\Delta c_{\pi} \propto \Delta \alpha_{\pi}$ $\propto \Delta C_{T}$ [Eqs. (11) and (12)] near the phase transition regime, once transition pressure $\pi_{t}$ and potential $\Psi_{t}$ increase nearly linear with temperature $T$. 


\section{CONCLUSIONS}

Our results reveal a thermal-mechano-electrical coupling in lipid monolayers. Using Einstein's theory of fluctuations in line with the experimental results, a linear relationship between electrical capacity, heat capacity, compressibility, and the thermal expansion coefficient has been found. These results enable us to calculate the heat capacity of lipid monolayers which has been shown to be within reasonable range with the heat capacity known from lipid vesicle suspensions. The relationship established in this work carries an enormous predictive power allowing to calculate heat and electrical properties from mechanical measurements. In future, it will be of enormous interest whether the same couplings hold for more complex or biological systems.

\section{ACKNOWLEDGMENTS}

M.F.S. likes to thank Dr. K. Kaufmann for stimulating discussions and lectures concerning the thermodynamic foundation of soft interfaces. Furthermore, we would like to acknowledge very helpful remarks from Professor Erich Sackmann. Financial support is acknowledged from the German government through the Cluster of Excellence "NIM" and the DFG (SPP 1313). M.F.S. and D.S. like to thank the Fond der Chemischen Industrie as well as the Elitenetzwerk Bayern (CompInt).
[1] H. Mohwald, Annu. Rev. Phys. Chem. 41, 441 (1990).

[2] E. Sackmann, Science 271, 43 (1996).

[3] T. Heimburg, Biochim. Biophys. Acta 1415, 147 (1998).

[4] L. D. Landau and E. M. Lifschitz, Course of Theoretical Physics (Butterworth-Heinemann, Frankfurt, 1987), Vol. 6.

[5] T. Shimojo and T. Ohnishi, J. Biochem. (Tokyo) 61, 89 (1967).

[6] H. Beitinger, V. Vogel, D. Möbius, and H. Rahmann, Biochim. Biophys. Acta 984, 293 (1989).

[7] V. Vogel and D. Möbius, J. Colloid Interface Sci. 126, 408 (1988).

[8] O. Albrecht, H. Gruler, and E. Sackmann, J. Phys. I 39, 301 (1978).

[9] H. Möhwald, Structure and Dynamics of Membranes (Elsevier, Amsterdam, 1995).

[10] M. Hato, H. Minamikawa, K. Tamada, T. Baba, and Y. Tanabe, Adv. Colloid Interface Sci. 80, 233 (1999).

[11] M. F. Schneider, G. Mathe, C. Gege, R. R. Schmidt, and M. Tanaka, J. Phys. Chem. B 105, 5178 (2001).
[12] B. Wunderlich, C. Leirer, A.-L. Idzko, U. F. Keyser, A. Wixforth, V. M. Myles, T. Heimburg, and M. F. Schneider, Biophys. J. 96, 4592 (2009).

[13] J. Griesbauer, A. Wixforth, and M. F. Schneider, Biophys. J. 97, 2710 (2009).

[14] V. V. Plotnikov, J. M. Brandts, L.-N. Lin, and J. F. Brandts, Anal. Biochem. 250, 237 (1997).

[15] E. Sackmann, in From Vesicles to Cells, edited by E. Sackmann and R. Lipowsky (Elsevier, Amsterdam, 1996), Vol. A.

[16] M. F. Schneider, D. Marsh, W. Jahn, B. Kloesgen, and T. Heimburg, Proc. Natl. Acad. Sci. U.S.A. 96, 14312 (1999).

[17] C. L. Leirer, B. Wunderlich, V. M. Myles, and M. F. Schneider, Biophys. Chem. 143, 106 (2009).

[18] C. L. Leirer, B. Wunderlich, A. Wixforth, and M. F. Schneider, Phys. Biol. 96, 6 (2009).

[19] T. Franke, C. L. Leirer, A. Wixforth, N. Dan, and M. F. Schneider, ChemPhysChem 10, 2852 (2009).

[20] O. G. Mouritsen, Biochim. Biophys. Acta 731, 217 (1983).

[21] O. G. Mouritsen, Chem. Phys. Lipids 57, 179 (1991). 